\title{
A Simplified Liquid Chromatography-Mass Spectrometry Method for Simultaneous Determination of Pyrimethamine, Sulphadoxineand Artesunate in Human Plasma
}

\author{
S M Sandhya, P S Shijikumar \\ ${ }^{1}$ Sree Krishna College of Pharmacy \& Research Centre, Parassala, Trivandrum, India. \\ ${ }^{2}$ Jamia Salafiya Pharmacy College, Pulikkal, Malappuram, India.
}

\section{ARTICLE INFO \\ Article history: \\ Received on: 05/03/2015 \\ Revised on: 23/03/2015 \\ Accepted on: 21/04/2015 \\ Available online: 27/06/2015}

\section{Key words:}

Pyrimethamine,

Sulphadoxine, Artesunate,

Artemether, LC-MS, Plasma,

Validation.

\begin{abstract}
This paper presents the development of a new LC-MS method for the determination of pyrimethamine, sulphadoxine and artesunate in human plasma. The analytes were extracted by liquid-liquid extraction with ethyl acetate. The analytes and internal standard artemether were separated on a Phenomenex $\mathrm{C}_{18}$ column using gradient elution with $20 \mathrm{mM}$ ammonium acetate (mobile phase A) and methanol (mobile phase B) both containing $0.5 \%$ acetic acid at a flow rate of $0.8 \mathrm{ml} / \mathrm{min}$. The calibration curves were constructed over the range of 5-30 $\mathrm{ng} / \mathrm{ml}$ for pyrimethamine, $50-300 \mathrm{ng} / \mathrm{ml}$ for sulphadoxine and $25-150 \mathrm{ng} / \mathrm{ml}$ for artesunate respectively. Within day and between-day precision and accuracy did not exceed $8 \%$. All the three analytes were found to be stable in plasma samples with no evidence of degradation during three freeze-thaw cycles and three months storage in -20 ${ }^{\circ} \mathrm{C}$. According to the validation results, the proposed method was found to be specific, accurate, precise and could be applied to the simultaneous quantitative analysis of pyrimethamine, sulphadoxine and artesunate in human plasma.
\end{abstract}

\section{INTRODUCTION}

Pyrimethamine (PYR; 5-(4-Cholorophenyl)-6-ethyl pyrimidine 2, 4-diamine; sulphadoxine (SPDX; 4-Amino-N-[5, 6-dimethoxy-4-pyrimidinyl] benzene sulphonamide; artesunate (ARTS; 3R, 5aS, 6R, 8aS, 9R, 10S, 12R, 12aR)-Decahydro3,6,9-trimethyl-3,12 epoxy-12 H-pyrano [4-3-j] 1,2benzodioxepin-10-ol hydrogen succinate (Figure 1) were used as antimalarial drugs. Synergism between PYR and SPDX is explained by inhibition of two steps in an essential metabolic pathway. The two steps involved are the utilization of p-amino benzoic acid for the synthesis of dihydropteroic acid, which is catalysed by dihydropteroate synthase and inhibited by SPDX and the reduction of dehydrofolate to tetrahydrofolate, which is catalysed by dihydrofolate reductase and inhibited by PYR (Risha et al, 2002; Srivastava et al, 2013). ARTS is a semisynthetic derivative of artemisinin, a naturally occurring sesquiterpene endoperoxide. It has been shown to be highly

* Corresponding Author

Email: sandhyashiji82@gmail.com active against multi-drug-resistant falciparum malaria in several regions dealing with high resistance rate against the classic antimalarials. ARTS have a rapid onset of therapeutic effect after extensive biotransformation of artesunate into dihydro artemisinin which is likely to provide beneficial therapeutic outcomes in severe or cerebral malaria. Due to the spread of multidrug-resistant P. falciparum malaria, artesunate plus sulphadoxinepyrimethamine is recommended as the first line treatment for uncomplicated P. falciparum malaria. This combination therapy remained effective against falciparum malaria despite common mutations promoting resistance to antifolate drugs (Day et al, 2007; Nosten et al, 2007). The reported methods for quantification of PYR and SPDX individually or in combination with other drugs from dosage form or in biological fluids include various analytical methods such as spectrophotometry (Onah et al, 2002; Meena et al, 2013), HPLC with UV (Green et al, 1995; AtemnKeng et al, 2007; Berzas et al, 2000; Eljaschewitsch et al, 1996; Astier et al, 1997; Bergqvist et al, 1985; Midskov et al, 1984; Edstein et al, 1984), HPLC with MS (Koesukwiwat et al, 2007), HPTLC (Meena et al, 2013), supercritical fluid chromatography (Bhoir et al, 2001) and spectrofluorimetry (Idowu et al, 1985). 
Reported HPLC detection systems for ARTS alone or in combination with other drugs include electrochemical detection (Gu et al, 2008; Zhou et al, 1987; Na-Bangchang et al, 1998; Navaratnam et al, 1997) derivatization and post-column degradation before UV detection (Zou et al, 1987; Batty et al, 1996; Taylor et al, 2000) and MS (Van Quekelbergh et al, 2008; Naik et al, 2005).

Although some reports have been published with regard to HPLC determination of PYR, SPDX and ARTS alone or in combination with other drugs, no data have been published for simultaneous determination of all three analytes. Here we describe a LC-MS method with a short sample preparation step for the analysis of PYR, SPDX, and ARTS in human plasma, capable of quantifying above three drugs using same procedure, which can substantially reduce the overall cost of the analysis. Also, an internal standard artemether was used which improves the precision of the assay.

\section{EXPERIMENTAL}

\section{Materials}

Pharmaceutical grade API, pyrimethamine and sulphadoxine were obtained as gift samples from M/s. Medopharm Pharmaceuticals, Karnataka, India. Artesunate and internal standard artemether were obtained from M/s. Zydus Cadila, Ahmadabad, India. HPLC grade methanol and analytical grade ammonium acetate, glacial acetic acid and ethyl acetate were procured from Merck Chemicals, Mumbai, India. Drug free (blank) heparinized human plasma was obtained from MIMS Hospital, Calicut, India and was stored at $-70^{\circ} \mathrm{C}$ prior to use.

\section{LC-MS System}

A Shimadzu LC-MS 2020 (Shimadzu, Japan) equipped with binary solvent delivery system, column compartment and photo diode array detector (PDA) was used for all analysis. The chromatographic separation was performed on Phenomenex $\mathrm{C}_{18}$ column (i.d. $250 \mathrm{~mm} \times 4.6 \mathrm{~mm}, 5 \mu \mathrm{m}$ ) and the column oven temperature was set at $30{ }^{\circ} \mathrm{C}$. The instrument was operated by switching electrospray ionization (ESI) source in positive and negative ionization modes in a single run. The ESI was performed using nitrogen gas to assist nebulization (flow rate was set at 1.5 $\mathrm{L} / \mathrm{min}$ ), capillary voltage at $1.6 \mathrm{kV}$ and temperatures of Curved Desolvation Line (CDL) and heat block at $250{ }^{\circ} \mathrm{C}$ and $300{ }^{\circ} \mathrm{C}$ were used. All instrumentation data were collected and synchronized by Lab solutions software (Version 7.1) from Shimadzu, Japan.

\section{Chromatographic conditions}

Reverse phase chromatographic separation was carried out on Phenomenex $\mathrm{C}_{18}$ column and all the four analytes including internal standard were resolved using a gradient elution of $20 \mathrm{mM}$ ammonium acetate and methanol both containing $0.5 \%$ acetic acid. The flow rate was $0.8 \mathrm{ml} / \mathrm{min}$ with an injection volume of $20 \mu \mathrm{L}$. The run time was $20 \mathrm{~min}$. Quantification was achieved by measurement of peak area ratios of analyte to internal standard.

\section{Standard solutions}

Solutions $(1 \mathrm{mg} / \mathrm{ml})$ of PYR, SPDX and ARTS were prepared separately in methanol. They were mixed and diluted with methanol to yield a stock solution containing $50 \mu \mathrm{g} / \mathrm{ml}$ of each analyte. This solution was then used to spike, blank plasma to give a series of calibration standards containing 5-30 ng/ml PYR, 50-300 ng/ml SPDX and 25-150 ng/ml ARTS. Low, medium and high concentration quality control samples containing each analyte were prepared independently using the same procedure. A $1 \mathrm{mg} / \mathrm{ml}$ stock solution of artemether was diluted suitably with methanol to give $100 \mathrm{ng} / \mathrm{ml}$ IS working solution. In each analytical run, calibration standards, quality control samples and unknown where treated together.

\section{Sample preparation}

Plasma samples stored at $-70{ }^{\circ} \mathrm{C}$ were allowed to thaw at room temperature and then subjected to liquid-liquid extraction (LLE). To $100 \mu \mathrm{L}$ plasma in a glass-stopped $10 \mathrm{ml}$ tube, $100 \mu \mathrm{L}$ IS working solution and $2 \mathrm{ml}$ HPLC grade ethyl acetate were added. The mixture was vortexed for $60 \mathrm{~s}$, centrifuged at $10,000 \times \mathrm{g}$ for 5 min and the upper organic layer transferred to another clean glass tube and evaporated to dryness at $40{ }^{\circ} \mathrm{C}$ with nitrogen. The dry residue obtained was reconstituted in $100 \mu \mathrm{L}$ of mobile phase and $20 \mu \mathrm{L}$ was injected to HPLC-MS.

\section{Assay validation}

The method was validated for specific, linearity, precision, accuracy, recovery, matrix effects and stability according to FDA guidelines (FDAUS, 2001).

\section{Linearity and specificity}

To construct the calibration curve, six replicates $(20 \mu \mathrm{L})$ of each standard solution were injected immediately after preparation into column and peak area of the chromatograms was measured. The peak area ratios of PYR, SPDX and ARTS to that of internal standard were plotted against the corresponding concentration to obtain the calibration graph. Blank human plasma samples from six different sources were used to evaluate specificity of the method. The same sources of plasma were spiked with PYR, SPDX, ARTS and ARTM to get a final concentration corresponding to LLOQ and responses (area) of components were estimated.

\section{Lower limit of quantification (LLOQ) and limit of detection (LOD)}

The LLOQ concentrations were selected as the lowest levels of calibration curves established during the analytical method validation. Back calculated values of calibration samples with a bias and CV\% below $\pm 20 \%$ enables to determine the LLOQ values. The LOD was determined as the concentration that produced a signal three times above the noise level of a blank preparation. 

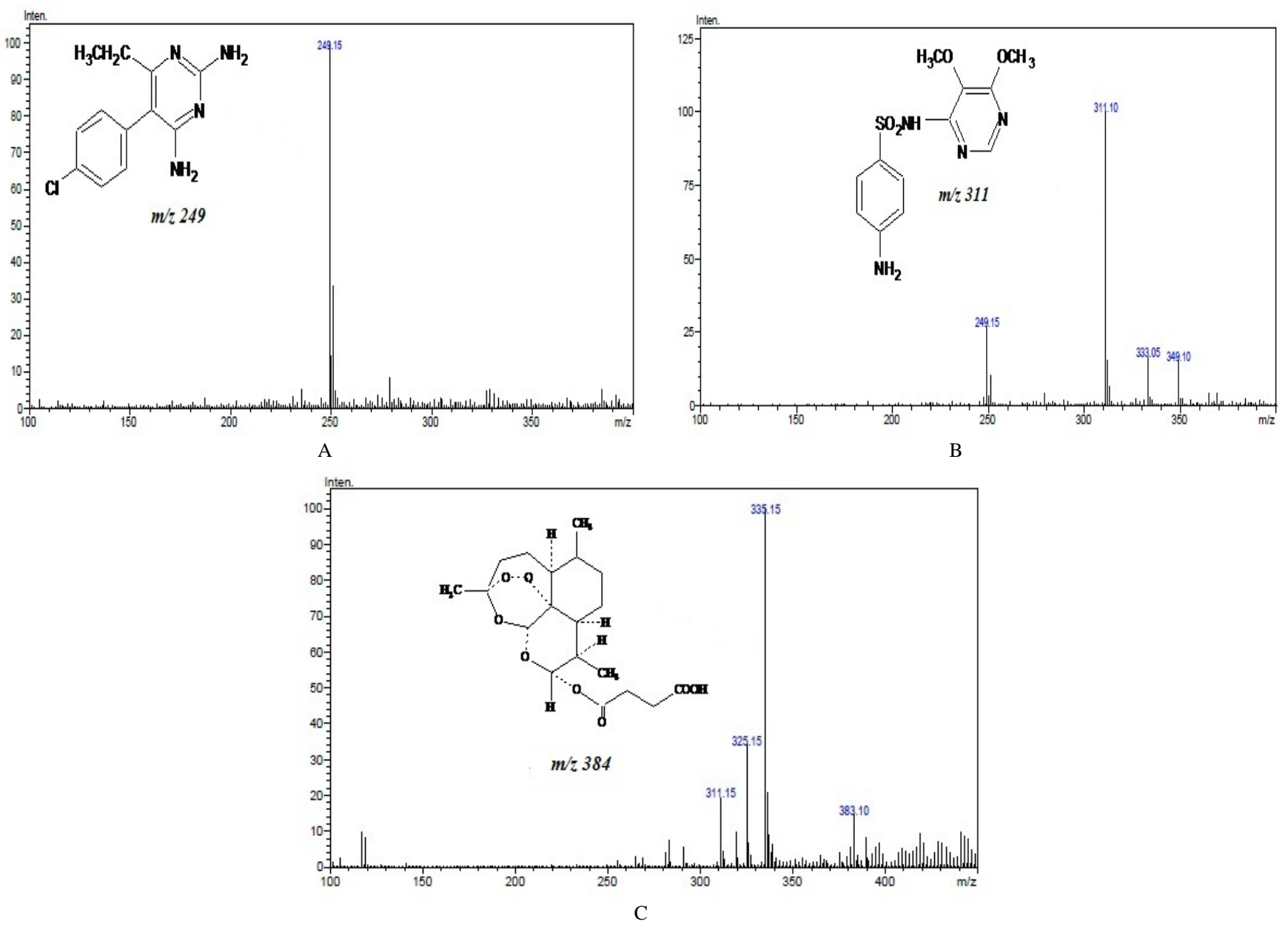

Fig. 1: Chemical structures and mass spectrum of PYR (A), SPDX (B) and (ARTS) C.

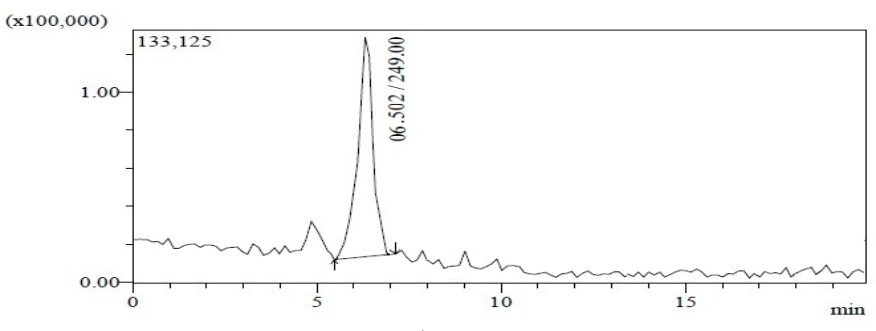

A

$(\mathrm{x} 1,000,000)$
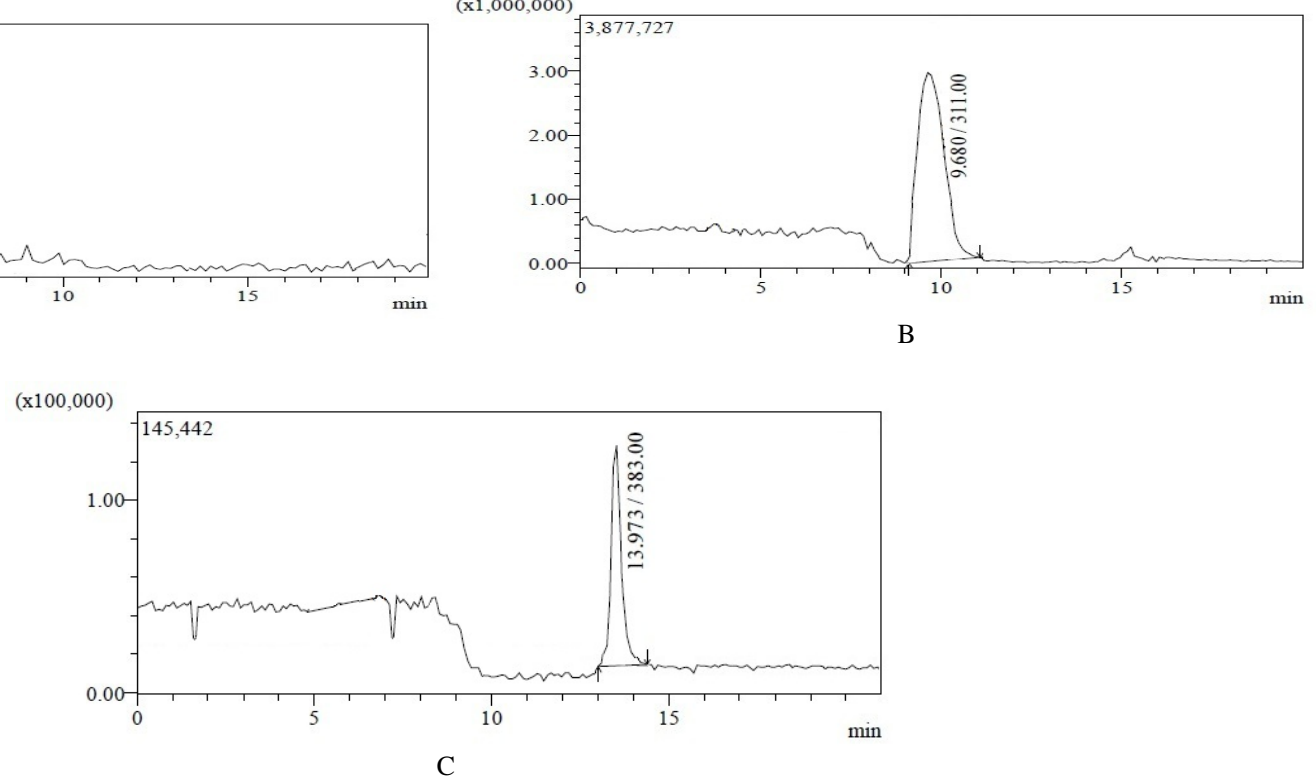

Fig. 2: Mass chromatogram of (A) PYR, (B) SPDX, and (C) ARTS in a single gradient run from MQC plasma sample. 


\section{Precision and accuracy}

For inter-day precision and accuracy, six replicates of each analyte's concentrations were injected over six consecutive days, whereas for intra-day precision six replicates of each analyte concentrations were injected on the same day. The six concentrations were chosen to encompass the whole range of calibration curve corresponding to the drug levels anticipated to occur in most of the samples. The precision was calculated as the coefficient of variation (CV \%). The recovery was assessed by comparing the peak area of six replicate analyses of plasma samples after treatment with drug solution of corresponding concentration.

\section{Stability study}

In freeze and thaw stability study, the analyte's stability was determined by storing at $-20{ }^{\circ} \mathrm{C}$ for $24 \mathrm{hrs}$, thawed at room temperature $\left(25^{\circ} \mathrm{C}\right)$ and finally refrozen three times. In short-term stability study, the lower and high concentrations of analytes were thawed unassisted at ambient room temperature of $25{ }^{\circ} \mathrm{C}$. In post preparative stability study, the analytes were assessed over anticipated run time of $8 \mathrm{hrs}$. In long-term stability study, three aliquots of lower and higher concentrations of each analyte was stored at $-20{ }^{\circ} \mathrm{C}$ over a period of three months.

\section{RESULTS AND DISCUSSION}

Developing an analytical method for all the three analytes in the same chromatographic run was a challenge, because the combination comprises both lipophilic drugs and structurally unrelated artemisinin derivative with distinct chromatographic behavior. Chromatographic separations using different analytical columns and mobile phase compositions were evaluated to optimize specificity and selectivity. After a series of screening experiments, it was concluded that, a Phenomenex $\mathrm{C}_{18}$ column and a mobile phase composition of $20 \mathrm{mM}$ ammonium acetate and methanol both containing $0.5 \%$ acetic acid gave good peak shape and a satisfactory retention time. Representative chromatograms extracted from spiked plasma with PYR, SPDX and ARTS at MQC (medium quality control) are shown in Figure 2, in which the retention times were 6.5, 9.6 and $13.9 \mathrm{~min}$ respectively. The effect of composition of mobile phase and flow rate on retention time of PYR, SPDX and ARTS was investigated. Similarly a concentration value of $20 \mathrm{mM}$ ammonium acetate was chosen for the optimum separation of analytes with well defined and resolved peaks. During optimization of ESI mode, the mass spectra for PYR, SPDX, ARTS and ARTM (IS) were characterized by peaks m/z 249, 311, 384 and 299 respectively (Figure 1).

A prominent feature of the present assay is efficient extraction of PYR, SPDX and ARTS from plasma matrix with the same extracting solvent system which renders the method more convenient and faster for bioequivalence studies of these drugs.

The method was found to be linear in the range of 5-30 $\mathrm{ng} / \mathrm{ml}$ for PYR, 50-300 $\mathrm{ng} / \mathrm{ml}$ for SPDX and 25-150 $\mathrm{ng} / \mathrm{ml}$ for ARTS respectively. Typical regression lines were found to be $\mathrm{y}=$ $0.401 \mathrm{x}+1.977(\mathrm{r}=0.9981), \mathrm{y}=0.039 \mathrm{x}+0.021(\mathrm{r}=0.9997)$ and $\mathrm{y}$ $=0.119 \mathrm{x}+0.050(\mathrm{r}=0.9987)$. Although blank plasma samples (with a zero concentration) were not selected in constructing the calibration curves, the $95 \%$ confidence interval of the intercept encompassed the origin. The minimum level at which the investigated compounds can be reliably detected (lower limit of detection, LLOD and quantified (lower limit of quantification, LLOQ) was determined experimentally. The concentrations 1.12 $\mathrm{ng} / \mathrm{ml}$ for PYR, $23.34 \mathrm{ng} / \mathrm{ml}$ for SPDX and $11.58 \mathrm{ng} / \mathrm{ml}$ for ARTS were considered as the lower limit of quantification, since the level is the lowest on the calibration curves and its concentration can be determined with acceptable precision (\% CV < 10) and accuracy $(\mathrm{SE}<15)$. The limits of detection for the three drugs examined were approximately $0.52 \mathrm{ng} / \mathrm{ml}, 9.31 \mathrm{ng} / \mathrm{ml}$ and $4.50 \mathrm{ng} / \mathrm{ml}$ for PYR, SPDX and ARTS respectively. As shown in Table 1, intraand inter-day precisions were respectively, < 6\% for PYR, < 5\% for SPDX and $<7 \%$ for ARTS respectively. Accuracy ranged from 84.21 to $94.82 \%$ for all the three analytes.

The results of stability study expressed as mean concentration $( \pm S D)$, determined at various storage conditions are summarized in Table 2. No tendency of degradation of PYR, SPDX or ARTS after storage at room temperature was observed. In three freeze-thaw cycles, all three compounds were stable in plasma, indicating no significant substance loss during repeated thawing and freezing.

Table 1: Recovery, accuracy and precision data $(n=6)$.

\begin{tabular}{|c|c|c|c|c|c|c|c|}
\hline \multirow[b]{2}{*}{ Analyte } & \multirow{2}{*}{$\begin{array}{l}\text { Sample } \\
\text { (ng/ml) }\end{array}$} & \multirow{2}{*}{$\begin{array}{l}\text { Concentration found } \\
(\text { Mean } \pm \text { SD; ng/ml) }\end{array}$} & \multirow[t]{2}{*}{ Mean recovery (\%) } & \multicolumn{2}{|c|}{ Intra-day } & \multicolumn{2}{|c|}{ Inter-day } \\
\hline & & & & $\begin{array}{c}\text { Precision } \\
\text { (CV \%) }\end{array}$ & Accuracy (\%) & $\begin{array}{c}\text { Precision } \\
\text { (CV \%) }\end{array}$ & $\begin{array}{c}\text { Accuracy } \\
(\%)\end{array}$ \\
\hline \multirow{3}{*}{ PYR } & 2 & $1.87 \pm 0.08$ & 93.5 & 4.27 & 93.9 & 5.23 & 90.86 \\
\hline & 15 & $14.40 \pm 0.42$ & 96.0 & 2.92 & 94.7 & 4.96 & 89.32 \\
\hline & 30 & $28.74 \pm 0.91$ & 95.8 & 3.16 & 94.5 & 2.98 & 84.21 \\
\hline \multirow{3}{*}{ SPDX } & 23 & $21.53 \pm 1.11$ & 93.6 & 5.16 & 92.6 & 3.58 & 91.56 \\
\hline & 150 & $142.87 \pm 5.66$ & 95.2 & 3.96 & 91.7 & 4.27 & 93.68 \\
\hline & 300 & $287.69 \pm 9.57$ & 95.9 & 3.34 & 94.8 & 3.97 & 94.08 \\
\hline \multirow{3}{*}{ ARTS } & 12 & $11.20 \pm 0.85$ & 93.3 & 7.59 & 94.5 & 5.41 & 89.54 \\
\hline & 75 & $72.58 \pm 2.54$ & 96.7 & 3.50 & 91.2 & 4.70 & 93.74 \\
\hline & 150 & $140.87 \pm 7.21$ & 93.9 & 5.12 & 93.7 & 6.51 & 94.75 \\
\hline
\end{tabular}


Table 2: Stability studies of PYR, SPDX and ARTS ( $\mathrm{n}=3$ ).

\begin{tabular}{|c|c|c|c|c|c|c|}
\hline \multirow{2}{*}{ Stability study } & \multicolumn{6}{|c|}{ Pyrimethamine concentration (ng/ml) } \\
\hline & \multicolumn{2}{|c|}{5} & \multicolumn{2}{|c|}{15} & \multicolumn{2}{|c|}{30} \\
\hline & Mean & $\%$ RSD & Mean & $\%$ RSD & Mean & $\%$ RSD \\
\hline Freeze and thaw & 4.51 & 4.85 & 14.01 & 2.78 & 28.64 & 3.67 \\
\hline Short-term & 4.62 & 2.34 & 14.32 & 4.20 & 28.57 & 4.98 \\
\hline Long-term & 4.23 & 2.35 & 15.02 & 2.67 & 29.04 & 3.55 \\
\hline \multirow[t]{4}{*}{ Post-preparative } & 4.80 & 4.88 & 14.01 & 2.58 & 29.54 & 5.67 \\
\hline & \multicolumn{6}{|c|}{ Sulphadoxine concentration (ng/ml) } \\
\hline & \multicolumn{2}{|c|}{$\mathbf{5 0}$} & \multicolumn{2}{|c|}{150} & \multicolumn{2}{|c|}{300} \\
\hline & Mean & $\%$ RSD & Mean & $\%$ RSD & Mean & $\%$ RSD \\
\hline Freeze and thaw & 47.87 & 3.57 & 147.57 & 4.12 & 294.37 & 4.10 \\
\hline Short-term & 48.04 & 2.35 & 145.87 & 4.64 & 291.76 & 3.87 \\
\hline Long-term & 47.85 & 3.25 & 145.87 & 3.47 & 293.67 & 4.21 \\
\hline \multirow[t]{4}{*}{ Post-preparative } & 48.22 & 2.74 & 147.80 & 3.28 & 295.18 & 3.32 \\
\hline & \multicolumn{6}{|c|}{ Artesunate concentration (ng/ml) } \\
\hline & \multicolumn{2}{|c|}{25} & \multicolumn{2}{|c|}{75} & \multicolumn{2}{|c|}{150} \\
\hline & Mean & $\%$ RSD & Mean & $\%$ RSD & Mean & $\%$ RSD \\
\hline Freeze and thaw & 23.45 & 2.89 & 72.58 & 3.27 & 143.87 & 4.45 \\
\hline Short-term & 24.01 & 2.40 & 72.06 & 1.98 & 145.21 & 5.87 \\
\hline Long-term & 23.87 & 2.36 & 74.02 & 3.29 & 147.51 & 4.04 \\
\hline Post-preparative & 24.75 & 1.54 & 73.61 & 3.97 & 146.34 & 3.38 \\
\hline
\end{tabular}

\section{CONCLUSION}

The present investigation describes a simple, sensitive and selective LC-MS method for analysis of PYR, SPDX and ARTS with LLE and mass spectroscopy detection. Ethyl acetate as extracting solvent gave excellent recovery for both drugs. The method was validated and met the requirement of linearity, recovery, accuracy and precision. Therefore this analytical procedure could readily be applied to routine analysis of plasma samples.

Conflicts of interest: None declared.

\section{REFERENCES}

Risha P G, Shewiyo D, Msami A, Masuki G, Vergote G, Vervaet C, Remon J P. In vitro evaluation of the quality of essential drugs on the Tanzanian market. Trop Med Int Health, 2002; 7(8):701-707.

Srivastava P, Ratha J, Shah N K, Mishra N, Anvikar A K, Sharma S K, Das M K, Srivastava B, Valecha N. A clinical and molecular study of artesunate + sulphadoxine-pyrimethamine in three districts of central and eastern India. Malar J, 2013; 12(1):247-252.

Day N, Dondrop A M. The management of patients with severe malaria. Am J Trop Med Hyg, 2007; 77(6):29-35.

Nosten F, White N J. Artemisinin-based combination treatment of falciparum malaria. Am J Trop Med Hyg, 2007; 77(6):181-192.

Onah J O, Odeiani J E. Simultaneous spectrophotometric determination of sulphadoxine and pyrimethamine in pharmaceutical formulations. J Pharm Biomed Anal, 2002; 30(3):851-857.

Meena S, Sandhya S M. Validated spectrophotometric methods for simultaneous analysis of pyrimethamine and sulphadoxine in pharmaceutical dosage forms. Asian J Pharm Clin Res, 2013; 6(3):121123.

Green M D, Mount D L, Todd G D. Determination of sulphadoxine concentrations in whole blood using $\mathrm{C}_{18}$ solid phase extraction, sodium dodecyl sulfate and dimethylaminocinnamaldehyde. Analyst, 1995; 120(10):2623-2626.

AtemnKeng M A, Chimanuka B, Plaizier-Vercamman J. Quality evaluation of chloroquine, quinine, sulphadoxine-pyrimethamine and proguanil formulations sold on the market in East Congo DR. Clin Pharm Ther, 2007; 32(2):123-132.
Berzas J J, Castaneda G, Guzman F J. Simultaneous determination of sulfamethazine and pyrimethamine by liquid chromatography. J Chromatogr A, 2000; 870(1-2):169-177.

Eljaschewitsch J, Padberg J, Schurmann D, Ruf B. High performance liquid chromatographic determination of pyrimethamine, dapsone, monoacetyldapsone, sulphadoxine, and N-acetylsulfadoxine after rapid solid-phase extraction. Ther Drug Monit, 1996; 18(5):592-597.

Astier H, Renard C, Cheminel V, Soares S, Mounier C, Peyron F, Chaulet J F. Simultaneous determination of pyrimethamine and sulphadoxine in human plasma by high-performance liquid chromatography after automated liquid-solid extraction. J Chromatogr B Biomed Sci Appl, 1997; 698(1-2):217-223.

Bergqvist Y, Eriksson M. Simultaneous determination of pyrimethamine and sulphadoxine in human plasma by high-performance liquid chromatography. Trans Royal Soc Trop Med Hyg, 1985; 79(3):297301.

Midskov C. High-performance liquid chromatographic assay of pyrimethamine, sulfadoxine and its $\mathrm{N}_{4}$-acetyl metabolite in serum and urine after ingestion of suldox. J Chromatogr, 1984; 308:217-227.

Edstein M. Quantification of antimalarial drugs: I. Simultaneous measurement of sulphadoxine, $\mathrm{N}_{4}$-acetyl sulphadoxine and pyrimethamine in human plasma. J Chromatogr, 1984; 305(2):502-507.

Koesukwiwat U, Jayenta S, Leepipatpiboon N. Validation of a liquid chromatography-mass spectrometry multi-residue method for the simultaneous determination of sulfonamides, tetracyclines, and pyrimethamine in milk. J Chromatogr A, 2007; 140(1-2):147-156.

Meena S, Sandhya S M. Validated HPTLC method for simultaneous analysis of pyrimethamine and sulphadoxine in pharmaceutical dosage forms. J Chemistry, 2013; 698490:1-6.

Bhoir S I, Bhoir I C, Bhagwat A M, Sundaresan M. Determination of sulfadoxine in human plasma using packed-column supercritical fluid chromatography. J Chromatogr B Biomed Sci Appl, 2001; 757(1):39-47.

Idowu O R, Dada O A. Simple and sensitive spectrofluorimetric determination of pyrimethamine in biological fluids. Anal Chim Acta, $1985 ; 169: 361-365$.

Gu Y, Li Q, Melendez V, Weina P. Comparison of HPLC with electrochemical detection and LC-MS/MS for the separation and validation of artesunate and dihydroartemisinin in animal and human plasma. J Chromatogr B Analyt Technol Biomed Life Sci, 2008; 67(2):213-218.

Zhou Z M, Anders J C, Chung H, Theoharides A D. Analysis of artesunic acid and dihydroqinghaosu in blood by high performance liquid 
chromatography with reductive electrochemical detection. J Chromatogr, 1987; 414(1):77-90.

Na-Bangchang K, Congpuong K, Hung LN, Molunto $\mathrm{P}$, Karbwang J. Simple high performance liquid chromatographic method with electrochemical detection for simultaneous determination of artesunate and dihydroartemisinin in biological fluids. J Chromatogr B Biomed Sci Appl, 1998; 708(1-2):201-207.

Navaratnam V, Mordi M N, Mansor S M. Simultaneous determination of artesunic acid and dihydroartemisinin in blood plasma by high-performance liquid chromatography for application in clinical pharmacological studies. J Chromatogr B Biomed Sci Appl, 1997; 692(1):157-162.

Zou A Q, Xie M, Luo X C. Determination of artesunic acid after chemical derivatization with o, p-nitrobenzyl N, N' diazopropyl isourea by HPLC with ultraviolet absorption. J Chromatogr, 1987; 410:217-221.

Batty K T, Davis T M, Thu L T, Binh T Q, Anh T K, Ilett K F. Selective high-performance liquid chromatographic determination of artesunate and $\alpha$ - and $\beta$-dihydroartemisinin in patients with falciparum malaria. J Chromatogr B Biomed Appl, 1996; 677(2):345-350.

Taylor R B, Awad M I, Reid R G, Moody R R. Determination of sodium artesunate in plasma using ion pairing high performance liquid chromatography. J Chromatogr B Biomed Sci Appl, 2000;744(2):415-421.
Van Quekelbergh S A, Soomro S A, Cordonnier J A, Jansen F H. Optimization of an LC-MS method for the determination of artesunate and dihydroartemisinin plasma levels using liquid-liquid extraction. J Anal Toxicol, 2008; 32(2):133-139.

Naik H, Murry D J, Kirsch L E, Fleckenstein L. Development and validation of high-performance liquid chromatography-mass spectroscopy assay for determination of artesunate and dihydroartemisinin in human plasma. J Chromatogr B Analyt Technol Biomed Life Sci, 2005; 816(1-2):233-242.

FDAUS, Guidance for Industry: Bioanalytical method validation. Rockville, MD, USA: Department of Health and Human Services, US-FDA/Center for Drug Evaluation and Research, 2001

\section{How to cite this article:}

Sandhya S M, Shiji kumar PS. A Simplified Liquid Chromatography-Mass Spectrometry Method for Simultaneous Determination of Pyrimethamine, Sulphadoxine and Artesunate in Human Plasma. J App Pharm Sci, 2015; 5 (06): 109-114. 